\title{
Parathyroid Imaging and Localization Using SPECT/CT: Initial Results
}

Ana Vaz ${ }^{1}$ and Marc Griffiths ${ }^{2}$

${ }^{I}$ Torbay Hospital, South Devon Health Care Foundation Trust, Torquay, United Kingdom; and ${ }^{2}$ University of the West of England, Bristol, United Kingdom

Primary hyperparathyroidism is caused by a single parathyroid adenoma in up to $90 \%$ of clinical cases. Selective surgical excision of the hyperfunctioning parathyroid gland is the treatment of choice for this condition, and parathyroid scintigraphy is one of the primary and standard methods used for preoperative localization. SPECT/CT is advantageous over planar imaging because SPECT/CT provides useful anatomic information, improving overall diagnostic confidence. This paper evaluates the initial findings of a hybrid parathyroid imaging technique that was put into effect after the installation of a SPECT/CT system in the nuclear medicine department at Torbay Hospital, Torquay, United Kingdom. The key findings from 3 of the initial sets of patient studies are discussed, along with the key learning points after the introduction of this new clinical service. Methods: From January 2009 until December 2009, 9 patients underwent a parathyroid study at Torbay Hospital (8 women [87.5\%] and 1 man [12.5\%]; mean age [ \pm SD], $61 \pm 10.12 \mathrm{y}$ ). All patients were referred from the endocrinology department because they had clinical signs of parathyroid adenoma $(67 \%)$ or required preoperative localization of parathyroid adenoma (33\%). For parathyroid scintigraphy, 640-990 $\mathrm{MBq}$ of $99 \mathrm{mTc}-$ sestamibi were administrated intravenously. A SPECT/CT system was used to acquire images from the level of the submandibular glands through the basal third of the heart. Three sets of images were acquired, and the processed images were reviewed for quality, attenuation correction, and registration. Results: Two (22\%) of the 9 studies were negative for possible adenoma, and 7 (78\%) were positive. Of the positive studies, $6(86 \%)$ showed an adenoma on the left inferior gland and only 1 (14\%) showed an adenoma in an eccentric position. Conclusion: The introduction of parathyroid SPECT/ CT has resulted in the development of new imaging and processing protocols within our nuclear medicine department. Introducing any new technique requires knowledge, understanding, and practical skills. The additional information gained from the parathyroid SPECT/CT technique has provided new skills for practitioners and increased reporting confidence for physicians.

\footnotetext{
Received Nov. 19, 2010; revision accepted Apr. 15, 2011

For correspondence or reprints contact: Marc Griffiths, Room 1E13, Glenside Campus, Faculty of Health and Life Sciences, University of the West of England, Bristol BS16 1DD, United Kingdom.

E-mail: marc.griffiths@uwe.ac.uk

Published online Jul. 27, 2011.

COPYRIGHT @ 2011 by the Society of Nuclear Medicine, Inc.
}

Key Words: SPECT/CT; parathyroid scintigraphy; adenoma; hyperparathyroidism

J Nucl Med Technol 2011; 39:195-200

DOI: 10.2967/jnmt.110.085522

$\mathbf{T}$ he emergence of transmission imaging technologies within hybrid imaging systems can be used in conjunction with SPECT techniques to help localize lesions identified within traditional nuclear medicine procedures. This approach has particular value when there are limited or no anatomic landmarks present on SPECT or when such landmarks are present but greater anatomic spatial resolution is required. The successful registration of SPECT with CT for localization purposes may aid definitive diagnosis and provide more precise lesion localization as part of surgical work-up for parathyroid adenomas. The parathyroid glands are small lentil-shaped glands that generally are located adjacent to the thyroid gland. Normal parathyroid glands measure approximately $6 \mathrm{~mm}$ in length, 3-4 $\mathrm{mm}$ in transverse diameter, and 1-2 $\mathrm{mm}$ in anteroposterior diameter and weigh $29.5 \mathrm{mg} \pm 17.8$ (mean $\pm \mathrm{SD}$ ) (1-4).

Normally, there are 2 superior and 2 inferior parathyroid glands located within the visceral space of the neck, posterior to the thyroid glands, near the tracheoesophageal groove. They may be either inside or outside the thyroid capsule $(1,2)$.

Ectopic inferior parathyroid glands may be located in the neck, inferior to the lower pole of thyroid lobe, either in the thyrothymic ligament or associated with the cervical portion of the thymus (26\%); on or adjacent to the posterior aspect of the middle third of the thyroid lobe $(7 \%)$; in the anterior mediastinum $(4 \%-5 \%)$; within the thyroid $(<3 \%)$; or along the carotid sheath $(<1 \%)(2)$.

Hyperparathyroidism occurs with a frequency of about 0.5 per 1,000 people, with approximately half the cases being asymptomatic and detected by serum calcium screening. Those with symptoms may have recurring low phosphate levels, weakness, fatigue, and bone pain (1-4).

Because of the small size and the location of the normal parathyroid glands, imaging with most modalities is dif- 
ficult. Between $80 \%$ and $85 \%$ of parathyroid adenomas are adjacent to the thyroid (5-7). The rest are ectopically placed and may be in the anterior or posterior superior mediastinum, within or next to the thymus, along the esophagus, along the carotid sheath, or even at the carotid bifurcation. This variation causes $5 \%$ of hyperfunctional parathyroid lesions to be missed at the initial surgical procedure, but hyperfunctioning glands can be imaged using nuclear medicine techniques $(1,3,8-14)$.

${ }^{99} \mathrm{~m}$ Tc-sestamibi is the radiopharmaceutical of choice for imaging hyperplastic parathyroid glands and adenomas because of its good energy characteristics for imaging and its avid localization in the mitochondria of parathyroid tissue. ${ }^{99 \mathrm{~m}} \mathrm{Tc}$-sestamibi has yielded sensitivity rates of about $90 \%$ in primary hyperparathyroidism $(1-3,15-28)$.

With this method, $900 \mathrm{MBq}$ are administered intravenously, and planar images are acquired at $15 \mathrm{~min}$ and at 1.0-3 $\mathrm{h}$ after injection. (1-3,15-28). ${ }^{99 \mathrm{~m}}$ Tc-sestamibi initially concentrates in normal thyroid tissue, thyroid adenomas, parathyroid adenomas, and hyperplastic parathyroid glands. Activity in the normal thyroid tissue significantly decreases with time. Thyroid adenomas and hyperplastic parathyroid glands initially have more intense activity than does the thyroid, and the intensity typically fades with time. Most parathyroid adenomas are more intense than is the thyroid on the early images and retain much of their activity on the delayed images, becoming more visible. Most parathyroid adenomas larger than $500 \mathrm{mg}$ can be detected with parathyroid scanning. Some thyroid adenomas may not fade as much as expected on delayed images, and some parathyroid adenomas may behave atypically by fading on delayed images. If delayed ${ }^{99 \mathrm{~m}} \mathrm{Tc}$-sestamibi images are inconclusive in either of these respects, it may be helpful to administer ${ }^{99 \mathrm{~m}} \mathrm{Tc}$-pertechnetate to see if the equivocal focus hyperconcentrates pertechnetate, as it will be likely to represent a thyroid adenoma $(17,18)$.

Parathyroid imaging is especially useful in patients with negative findings on neck exploration and recurrent or persistent hypercalcemia, but the technique is still unable to image hyperplastic or normal parathyroid glands consistently. The use of SPECT increases the sensitivity of planar examinations because it allows 3-dimensional imaging of the parathyroid gland. Especially when combined with CT, SPECT becomes particularly helpful for preoperative localization of eutopic and ectopic parathyroid adenomas (1-3,15-28).

Many surgeons routinely use ultrasound, radionuclide parathyroid imaging, or both before surgery, and scintigraphy is also useful for locating the adenoma during surgery $(1-3,15-28)$.

The primary goal of image interpretation is to detect and localize the hyperfunctioning gland. A diagnosis of parathyroid adenoma is confirmed by inspecting both the early images and the delayed images (Fig. 1). The scintigraphic patterns observed on early-phase images depend on the relative anatomic positions and radiopharmaceutical uptake of the parathyroid and thyroid glands $(15,16,20,22,27)$.

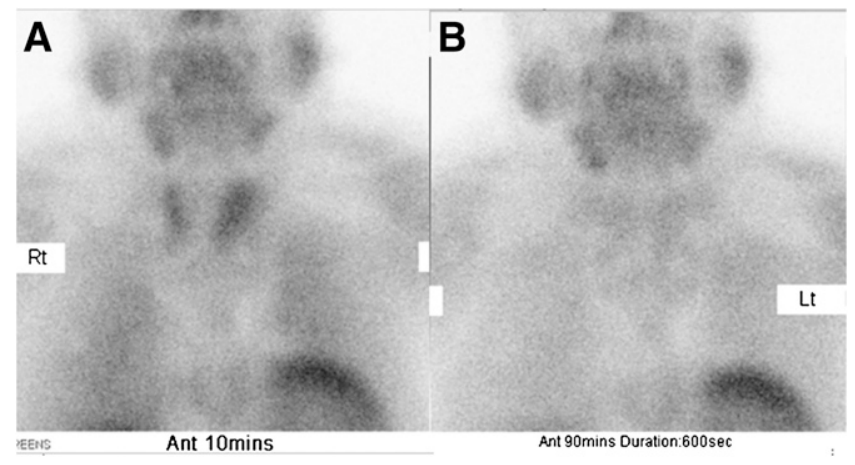

FIGURE 1. Early phase (A) and delayed phase (B) of planar 99mTc-sestamibi scan showing normal parathyroid findings.

Additional information may be obtained from the extent of radiopharmaceutical washout from the thyroid and parathyroid glands on delayed SPECT/CT images. There are 2 patterns of washout from the parathyroid glands: delayed washout (parathyroid gland retention of radiopharmaceutical on delayed images, usually accompanied by normal washout from the thyroid) and early washout (minimal or no retention of the radiotracer in the parathyroid gland on delayed-phase images; less common, although not rare) $(20,22,27)$.

Although the superior or inferior origin of a parathyroid gland often can be determined by its location, an enlarged gland may migrate and be seen in scintigraphic images at the level of the thyroid gland $(1-3,15-28)$.

The most frequent cause of a false-positive finding of a hyperfunctioning parathyroid gland on scintigraphy is the solid nodule that occurs in a solitary thyroid adenoma or a multinodular goiter (Fig. 2). Benign or malignant tumors that take up the radiopharmaceutical and result in falsepositive findings include breast, lung, and head and neck carcinomas and their lymph node and osseous metastases, as well as bronchial carcinoids. Within the neck, delayed washout of ${ }^{99} \mathrm{~m}$ Tc-sestamibi has been described in differentiated thyroid malignancies, primary thyroid lymphomas, cervical lymph node metastasis from papillary thyroid carcinoma, reactive lymph nodes, a remnant thymus, a parathyroid hormone-secreting paraganglioma, and an enlarged submandibular salivary gland. However, in the clinical set-
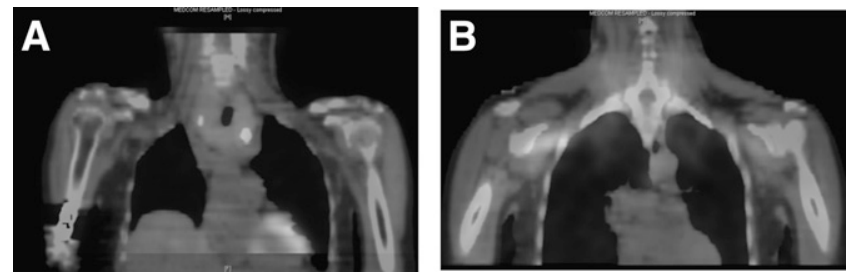

FIGURE 2. (A) SPECT/CT image showing possible adenoma, but thyroid uptake creates difficulty. (B) Misregistration, which may have resulted from patient movement between SPECT and CT acquisitions. This study was subsequently reprocessed. A color version of this figure is available as a supplemental file at http://tech.snmjournals.org. 
TABLE 1

Patient Data

\begin{tabular}{ccccc}
\hline Sex & Age $(\mathrm{y})$ & Reason for scan & Activity administered (MBq) & Results \\
\hline F & 66 & Preoperative localization & 952 & Parathyroid adenoma in left inferior gland \\
M & 65 & Clinical signs of adenoma & 955 & No evidence of functioning parathyroid adenoma \\
F & 49 & Clinical signs of adenoma & 719 & Parathyroid adenoma in left inferior gland \\
F & 53 & Preoperative localization & 899 & No evidence of functioning parathyroid adenoma \\
F & 56 & Clinical signs of adenoma & 899 & Parathyroid adenoma in left inferior gland \\
F & 60 & Preoperative localization & 880 & Parathyroid adenoma in eccentric position \\
F & 79 & Clinical signs of adenoma & 770 & Possible parathyroid adenoma in left inferior gland \\
F & 71 & Clinical signs of adenoma & 755 & Possible parathyroid adenoma in left inferior gland \\
F & 50 & Clinical signs of adenoma & 742 & Possible parathyroid adenoma in left inferior gland \\
\hline
\end{tabular}

ting of hyperparathyroidism, false-positive findings are uncommon $(1,20-22)$.

The factor most commonly reported to correlate with false-negative findings is the size of the parathyroid gland (i.e., smaller glands are less likely to be detected than larger glands $(16,20,22))$, and inclusion of anatomic information may benefit patient treatment. SPECT/CT is particularly useful for equivocal cases or when uniglandular disease has been undetected with ultrasound techniques $(29,30)$.

The objectives of this study were to collect the data of all patients who underwent a parathyroid study at Torbay Hospital, Torquay, United Kingdom, in 2009; list all relevant information; discuss the results; and highlight the key aspects of introducing parathyroid SPECT/CT as a new clinical service.

\section{MATERIALS AND METHODS}

Between January 2009 and December 2009, 9 patients underwent parathyroid scintigraphy in the Nuclear Medicine Department at Torbay Hospital. The patients included 8 women $(87.5 \%)$ and 1 man $(12.5 \%)$ and had a mean age $( \pm \mathrm{SD})$ of $61 \pm 10.12 \mathrm{y}$ (Table 1). All were referred by the endocrinology department for investigation of clinical signs of parathyroid adenoma (67\%) or for preoperative localization of parathyroid adenoma (33\%). Appropriate ethical approval was obtained from the department to access patients' information, acquired data, study results, and sample images. All patients consented to having their data used for research or training purposes.

For parathyroid scintigraphy, the patients received ${ }^{99 \mathrm{~m} T c-}$ sestamibi intravenously (mean dose, $815 \mathrm{MBq}$; range, 640990 MBq). A SPECT/CT system (Symbia T2; Siemens) was used to acquire images from the level of the submandibular glands through the basal third of the heart. Three sets of images were acquired, the first at $10 \mathrm{~min}$ after injection (a 600 -s static image with a $256 \times 256$ matrix and a zoom factor of 2.0) and the second at $90 \mathrm{~min}$ after injection (same parameters as before), followed by a SPECT/CT study.

The SPECT data acquisition comprised counts from the $15 \%$ energy windows at $140 \mathrm{keV}$, acquired into a $128 \times 128$ matrix, leading to a pixel size of $4.6 \times 4.6 \mathrm{~mm}$. A total of 64 frames, $30 \mathrm{~s}$ per frame, was acquired over a $360^{\circ}$ orbit. The camera heads were configured with high-resolution lowenergy parallel-hole collimators. Reconstruction was performed iteratively using 3-dimensional ordered-subsets expectation maximization with 4 iterations and 8 subsets. Images were smoothed with a 3-dimensional spatial gaussian filter (10 $\mathrm{mm}$ in full width at half maximum).

The CT parameters included a $130-\mathrm{kVp}$ tube, a $0.8-\mathrm{s}$ rotation time, and a $2 \times 2.5$ collimation. An image reconstruction algorithm (B80 reconstruction kernel) was applied, resulting in images with a slice thickness of $3 \mathrm{~mm}$ for a $1.5-\mathrm{mm}$ reconstruction increment. The processed SPECT and CT images were reviewed for adequacy of quality, attenuation correction, registration, and fusion.

\section{RESULTS}

Two (22\%) of the 9 studies were negative for possible adenoma, and $7(78 \%)$ were positive. Of the positive studies, $6(86 \%)$ showed an adenoma on the left inferior gland and only $1(14 \%)$ showed an adenoma in an eccentric position.

SPECT/CT was performed in only 8 of the 9 examinations because the washout images of 1 patient were unambiguous about the presence of adenoma (Fig. 3).

The following 3 cases are examples from the study population.

Case 1, a 66-y-old woman, presented with hypercalcemia possibly secondary to primary hyperparathyroidism. The endocrinology department had requested a parathyroid scan to assess parathyroid function before surgery. A $952-\mathrm{MBq}$

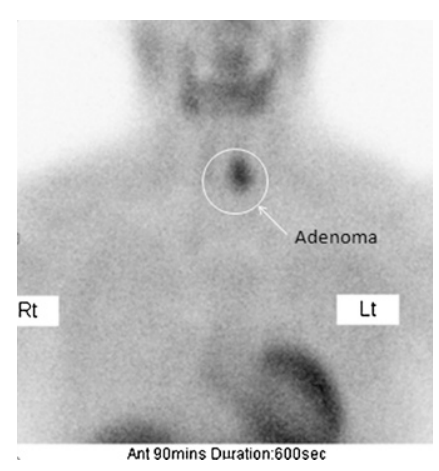

FIGURE 3. Adenoma in left inferior gland, visible in delayed phase of planar 99mTc-sestamibi scan. 


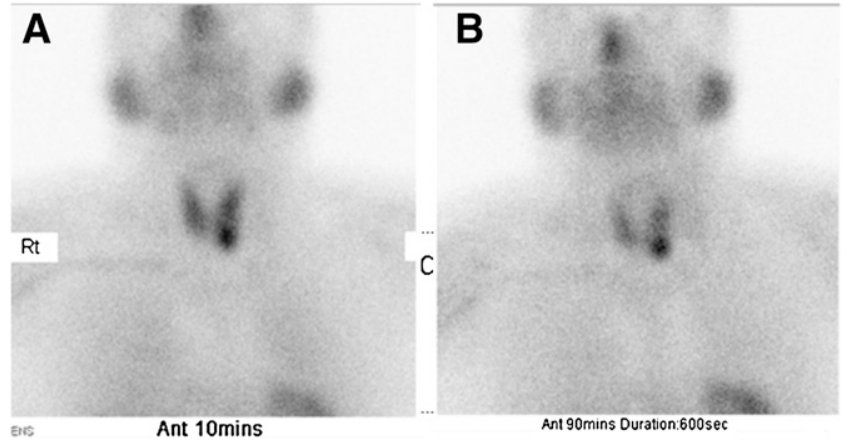

FIGURE 4. Early phase (A) and delayed phase (B) of planar $99 \mathrm{~m}$ Tc-sestamibi scan showing high uptake in parathyroid, consistent with adenoma.

dose of ${ }^{99 \mathrm{~m}} \mathrm{Tc}$-sestamibi was intravenously administered before the examination. The scan helped to confirm the presence of a parathyroid adenoma in the left inferior gland (Figs. 4 and 5).

Case 2, a 49-y-old woman, was referred to investigate possible hyperparathyroidism. She was injected with $719 \mathrm{MBq}$ of ${ }^{99 \mathrm{~m} T c-s e s t a m i b i . ~ F o c a l ~ i n c r e a s e d ~ u p t a k e, ~ p e r s i s t i n g ~ o n ~}$ the delayed images, was seen in the inferior aspect of the left thyroid lobe (Fig. 6). A corresponding finding could be seen posteriorly on the CT images (Fig. 7). The results were in keeping with a parathyroid adenoma.

Case 3, a 71-y-old woman, had presented to the endocrinology department with a clinically multinodular goiter and primary hyperparathyroidism. The scan had been requested to assess the dominant gland and to assess thyroid uptake. The patient was injected with $755 \mathrm{MBq}$ of ${ }^{99 \mathrm{~m} T c-}$ sestamibi before undergoing scanning. The scan showed the thyroid to be enlarged and heterogeneous, making assess-

FIGURE 5. Transverse (A), fused coronal (B), and fused sagittal (C) SPECT/CT images showing higher uptake in neck, consistent with parathyroid adenoma, which helped to support information given by planar scan. A color version of this figure is available as a supplemental file at http:// tech.snmjournals.org.
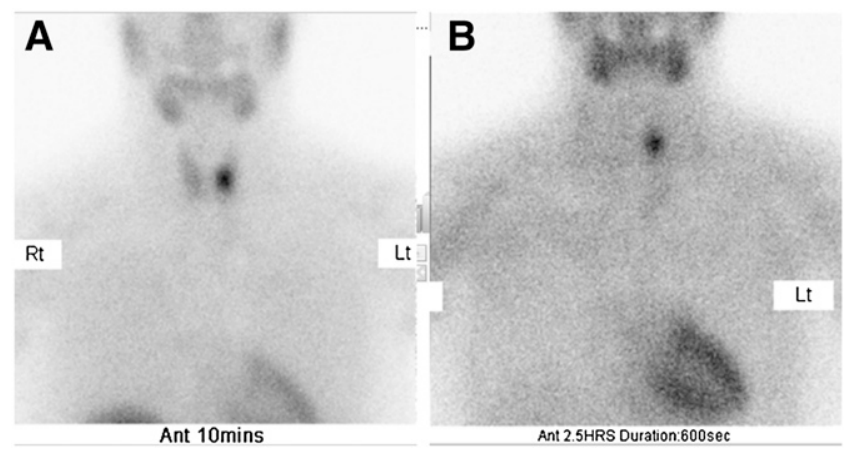

FIGURE 6. Early phase (A) and delayed phase (B) of planar 99mTc-sestamibi scan showing high-uptake area on left side of neck consistent with parathyroid adenoma.

ment for a parathyroid adenoma difficult. A focus of slightly more intense uptake that was seen posterior to the lower pole of the left lobe of the thyroid could equally have represented either a parathyroid adenoma or a thyroid nodule (Figs. 8 and 9). Because the scan was ambiguous, the patient was then referred for a thyroid scan with ${ }^{99 \mathrm{~m}} \mathrm{Tc}-$ pertechnetate. For this scan, $70 \mathrm{MBq}$ of ${ }^{99} \mathrm{~m} \mathrm{TcO}_{4}{ }^{-}$were administered intravenously, followed by a planar study. The thyroid gland seemed to be enlarged and contained uneven nodular uptake but no dominant nodule (Fig. 10).

\section{DISCUSSION}

SPECT/CT in the management of parathyroid adenomas has the potential to improve accuracy rates and further inform subsequent surgical decisions (30). The introduction of a new hybrid imaging system and the development of a new clinical service has created opportunities for the practitioners to learn new skills, which include optimal and safe use of transmission imaging techniques, CT-based processing algorithms, patient-positioning considerations for hybrid imaging, and personal dose monitoring. Introducing new technology and techniques within an established clinical environment can affect workforce dynamics and efficiency rates $(31)$.

The inclusion of CT information provides operators with new skills and techniques while applying attenuation correction values to the emission data. SPECT/CT also
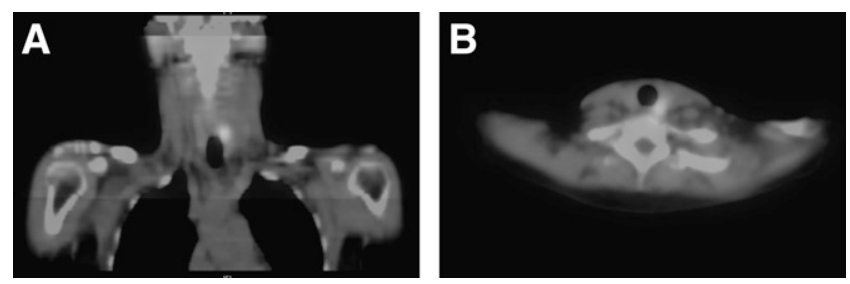

FIGURE 7. In same patient as in Figure 6, SPECT/CT coronal (A) and transverse (B) images that helped to confirm position of adenoma. Both images show high-uptake area consistent with parathyroid adenoma. A color version of this figure is available as a supplemental file at http://tech.snmjournals.org. 

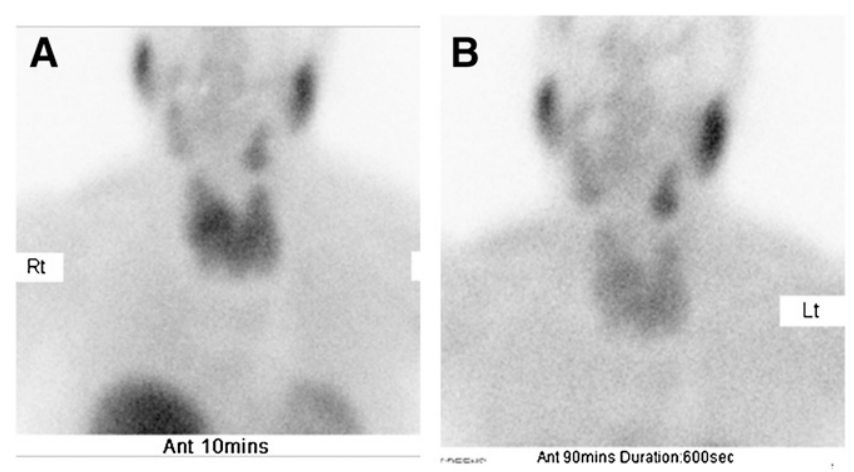

FIGURE 8. Early phase (A) and delayed phase (B) of planar $99 \mathrm{mTc}$-sestamibi scan showing irregular uptake within thyroid gland.

allows physicians to view and manipulate the data in 3 dimensions (30). Physicians have also reevaluated the reviewing and reporting of parathyroid images, to include the additional SPECT/CT datasets. Taking care that the appropriate look-up tables and $\alpha$-blending techniques are used with SPECT/CT is essential to ensure optimal identification of diseases. Protocols for the processing and secondary capturing of processed data are also essential for archiving and optimal retrieval from central or local PACS environments.

The additional time required for SPECT/CT examinations should also be factored in when a new imaging service in introduced. Clinical departments with limited access to SPECT/CT systems are required to carefully organize the imaging work flow, ensuring that centralized patient waiting areas do not become controlled radiation environments.

Potential limitations of this study include patient conditions (e.g., tissue attenuation due to a high body mass
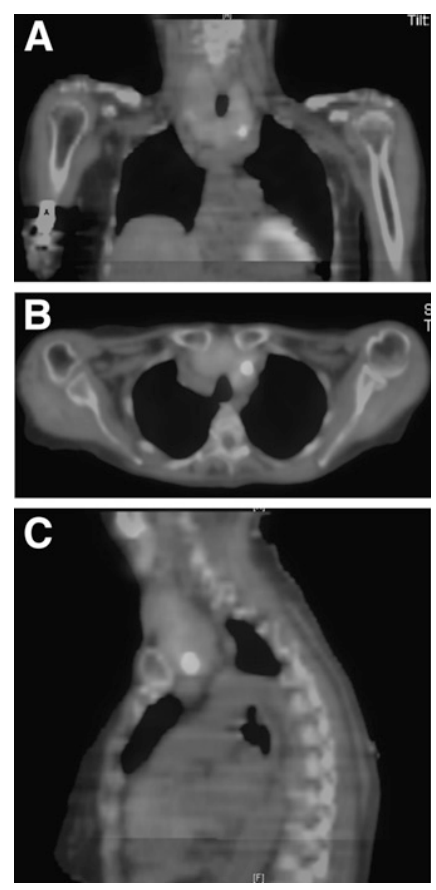

FIGURE 9. Coronal (A), transverse $(B)$, and sagittal $(C)$ SPECT/CT images showing uptake within neck area. Even though fused images helped with anatomic localization, disease within parathyroid gland could not be excluded. A color version of this figure is available as a supplemental file at http://tech.snmjournals. org.

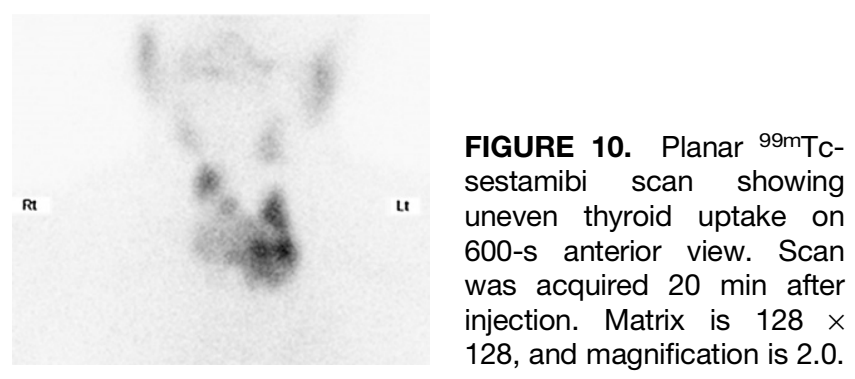

index), injection technique (extravasation of radiopharmaceutical), and scanning technique (e.g., lack of patient communication, bad positioning, and inaccurate processing techniques). In addition, there were different protocols in place within the department, leading to administered activities ranging between 640 and $990 \mathrm{MBq}$. Finally, this was an initial evaluation of the clinical value of SPECT/CT, and we acknowledge that the sample population was relatively small $(n=9)$. A future study should include a larger population and a higher number of male patients. However, published research indicates that the incidence of parathyroid adenoma is higher in women than men and also increases with age $(30,32)$.

\section{CONCLUSION}

This initial study demonstrated the clinical value of $\mathrm{SPECT} / \mathrm{CT}$ in the management of parathyroid patients. Using SPECT/CT requires additional imaging time and, therefore, appropriate planning and organization. Consideration is also required for the level of CT used during the SPECT/CT data acquisition. Our SPECT/CT system consists of a 2-slice CT unit and allows for dose modulation. The CT data are used only for attenuation correction and localization, not for diagnosis. However, we realize that other clinical departments may use CT data for diagnostic purposes.

Using SPECT/CT has improved the overall accuracy rates of parathyroid examination in our clinical department, and undertaking this initial study has provided an opportunity to evaluate our current services for parathyroid referrals. Streamlining the procedural and processing aspects of this particular examination has also enabled the department workforce to offer greater flexibility and gain a better understanding of the optimal acquisition and processing parameters.

\section{ACKNOWLEDGMENT}

No potential conflict of interest relevant to this article was reported.

\section{REFERENCES}

1. Mariani G, Gulec SA, Rubello D. Preoperative localization and radioguided parathyroid surgery. J Nucl Med. 2003;44:1443-1458. 
2. Rubello D, Pelizzo MR, Boni G. Radioguided surgery of primary hyperparathyroidism using the low ${ }^{99 \mathrm{~m}} \mathrm{Tc}$-sestamibi dose protocol: multi-institutional experience from the Italian Study Group on Radioguided Surgery and ImmunoScintigraphy (GISCRIS). J Nucl Med. 2005;46:220-226.

3. Rubello D, Gross MD, Mariani G, Al-Nahhal A. Scintigraphic techniques in hyper-parathyroidism: from pre-operative localization to intra-operative imaging. Eur J Nucl Med Mol Imaging. 2007;34:926-933.

4. Billotey C, Sarfati E, Aurengo A. Advantages of SPECT in technetium-99msestamibi parathyroid scintigraphy. J Nucl Med. 1996;37:1773-1778.

5. Pattou F, Huglo D, Proye C. Radionuclide scanning in parathyroid disease. $\mathrm{Br} \mathrm{J}$ Surg. 1998;85:1605-1616.

6. Francis IS, Loney E, Buscombe JR, Thakrar DS, Berger L, Hilson AJW. 99TcmMIBI dual-phase SPET imaging: concordance with ultrasound. Nucl Med Commun. 1999;20:487-488.

7. Loney EL, Buscombe JR, Hilson AJW, Davenport A, Francis IS. Preoperative imaging of parathyroid glands. Lancet. 1999;354:1819-1820.

8. Moka D, Voth E, Dietlien M, Larean-Avellanda A, Schicha H. Technetium 99mMIBI-SPECT: a highly sensitive diagnostic tool for localization of parathyroid adenomas. Surgery. 2000;128:29-35.

9. O'Doherty MJ, Kettle AG. Parathyroid imaging: preoperative localization. Nucl Med Commun. 2003;24:125-131.

10. Spanu A, Falchi A, Manca A. The usefulness of neck pinhole SPECT as a complementary tool to planar scintigraphy in primary and secondary hyperparathyroidism. J Nucl Med. 2004;45:40-48.

11. Rubello D, Massarro A, Cittadin S. Role of ${ }^{99 \mathrm{~m} T c-s e s t a m i b i ~ S P E C T ~ i n ~ a c c u r a t e ~}$ selection of primary hyperparathyroid patients for minimally invasive radioguided surgery. Eur J Nucl Med Mol Imaging. 2006;33:1091-1094.

12. Gayed IW, Kim EE, Broussard WF. The value of ${ }^{99 \mathrm{~m}} \mathrm{Tc}$-sestamibi SPECT/CT over conventional SPECT in the evaluation of parathyroid adenomas or hyperplasia. J Nucl Med. 2005;46:248-252.

13. Krausz Y, Bettman L, Guranlki L. Technetium-99m-MIBI SPECT/CT in primary hyperparathyroidism. World J Surg. 2006;30:76-83.

14. Serra A, Bolasco P, Satta L. Role of SPECT/CT in the preoperative assessment of hyperparathyroid patients. Radiol Med. 2006;111:999-1008.

15. Merrick M. Essentials of Nuclear Medicine. 2nd ed. London, U.K.: Springer Publications; 1998:112-146.

16. Perkins AC. Nuclear Medicine: Science and Safety. 3rd ed. London, U.K.: John Libbey and Company Ltd.; 1996: 94-145.
17. Ell PJ, Gambhir SS. Nuclear Medicine in Clinical Diagnosis and Treatment. 3rd ed. London, U.K.: Churchill Livingstone; 2004.

18. Saha GB. Fundamentals of Nuclear Pharmacy. 4th ed. New York, NY: Springer; 1997.

19. Powsner RP, Powsner ER. Essential Nuclear Medicine Imaging Physics. 4th ed. New York, NY: Blackwell Publishing; 2006:136-151.

20. Thrall J, Ziessman H. Medicina Nuclear. 2nd ed. Rio de Janeiro: Guanabara Koogan S.A.; 2003:28-31.

21. Grenier PR. Performance of scintillation cameras [abstract]. J Nucl Med. 1998;17(suppl):665P

22. Júnior FAM, Guiberteau MJ. Essentials of Nuclear Medicine Imaging. 5th ed. London, U.K.: Saunders Elsevier; 2006:85-181.

23. Cherry RS, Sorenson JA, Phelps ME. Physics in Nuclear Medicine. 3rd ed. New York, NY: Saunders; 2003:195-197.

24. Pretorius PH, Vanrensburg AJ, Witter MG, Serfontein DE, Herbst CP. The channel ratio method of scatter correction for radionuclide image quantitation. $\mathrm{J} \mathrm{Nucl}$ Med. 1993;34:330-335.

25. Hobbie RK. Intermediate Physics for Medicine and Biology. 4th ed. New York, NY: Struders; 1997:145-158.

26. Society of Nuclear Medicine procedure guideline for parathyroid scintigraphy. SNM Web site. Available at: http://interactive.snm.org/docs/Parathyroid_v3.0. pdf. Accessed May 19, 2011.

27. Murray IPC, Ell PJ. Nuclear Medicine in Clinical Diagnosis and Treatment. 2nd ed. London, U.K.: Churchill Livingstone; 1998:45-118.

28. Cherry RS, Sorenson JA, Phelps ME. Physics in Nuclear Medicine. 3rd ed. New York, NY: Saunders; 2005:170-187.

29. Tublin ME, Pryma D, Yim J, Ogilvie J, Mountz J, Bencherif B. Localization of parathyroid adenomas by sonography and technetium tc $99 \mathrm{~m}$ sestamibi single-photon emission computed tomography before minimally invasive parathyroidectomy: are both studies really needed? J Ultrasound Med. 2009;28: 183-190.

30. Eslamy HK, Ziessman H. Parathyroid scintigraphy in patients with primary hyperparathyroidism: ${ }^{99 \mathrm{~m}} \mathrm{Tc}$ sestamibi SPECT and SPECT/CT. Radiographics. 2008;28:1461-1476

31. Larsson W, Aspelin P, Bergquist M, et al. The effects of PACS on radiographer's work practice. Radiography. 2007;13:235-240.

32. Bäcklund M, Grander D, Brandt L, Hall P, Ekbom A. Parathyroid adenoma and primary CNS tumors. Int J Cancer. 2005;113:866-869. 